\title{
iWatch: A Fall and Activity Recognition System Using Smart Devices
}

\author{
Sittichai Sukreep ${ }^{1 *}$, Khalid Elgazzar ${ }^{2}$, Henry $\mathrm{Chu}^{2}$, Pornchai Mongkolnam ${ }^{1}$, Chakarida \\ Nukoolkit ${ }^{1}$ \\ ${ }^{1}$ School of Information Technology, King Mongkut's University of Technology Thonburi, Bangkok, Thailand. \\ ${ }^{2}$ School of Computing \& Informatics, University of Louisiana at Lafayette, Louisiana, USA. \\ * Corresponding author. Email: sittichai.s@mail.kmutt.ac.th \\ Manuscript submitted August 15, 2018; accepted November 5, 2018. \\ doi: 10.17706/ijcce.2019.8.1.18-31
}

\begin{abstract}
Recent reports show that the average life expectancy is increasing worldwide, posing significant overhead on healthcare systems and increasing demands on long-term care facilities. One of the grand challenges directly related to growing ageing societies is the implications of falling. Many elderly people live alone, especially those in Western countries who cannot afford living in a senior house or retirement facility. In such cases, not only falling is a major concern, but also daily activities must be continuously monitored and analyzed to provide immediate support when needed. Vital signs and environment context are also crucial conditions for pre- and post-event assessments. Thanks to technology advancements and widespread adoption of the Internet of Things which enables us to provide smart and ubiquitous healthcare services. In this paper, we propose iWatch, a smart and flexible system for fall detection and activity recognition using common smart devices, a smartwatch and a smartphone. Machine learning techniques are used to build efficient and highly accurate activity recognition classifiers. iWatch also provides health risk analysis using threshold-based models and leverages visualization tools to better communicate with the user. iWatch is a promising technology that provides a small step in a giant leap to revolutionize healthcare services, especially for those who needs extra care.
\end{abstract}

Key words: Health monitoring, smartwatch, IoT, daily activity, fall.

\section{Introduction}

The average life expectancy is increasing worldwide due to improved healthcare. This not only creates overhead on the healthcare system, but also poses many challenges associated with growing ageing societies [1], especially for those who live alone. Falling is one of the primary risks associated with aging that causes significant health problems, severe injuries and possibly deaths [2]. Slip and falling injuries could be fatal without getting immediate medical assistance [3]. It is necessary to ensure the safety of our loved ones and be able to receive immediate notifications if something goes wrong.

Several remote health monitoring systems have been developed, whether to reduce the overhead on the healthcare infrastructure and long-term care facilities or to provide better convenience to patients how need contiguous monitoring. However, currently there is a lack of robust proofs regarding user convenience, outdoor usability and cost efficiency. Here it comes mobile computing and smart devices to offer convenient healthcare monitoring to patients at a very low cost and high ubiquity [4]. Towards this end, we propose, 
iWatch, a robust and comprehensive activity recognition system to support remote healthcare monitoring. iWatch leverages machine learning techniques to detect falling and recognize a number of important activities to assist elderly to live a healthier and more independent life. iWatch is an affordable, unobtrusive, user-friendly, and highly accurate system that fits both indoor and outdoor.

The major contributions in this paper are summarized as follows.

- A holistic cost-efficient health monitoring system for indoor and outdoor settings.

- A highly accurate daily activity and fall detection algorithm.

- An easy to understand timeline tracking of the user activity using visualization techniques.

\section{Related Worked}

Many researchers have addressed the challenges of activities recognition and fall detection using different devices, techniques and visualizations tools. In the following, we highlight some of the previous research efforts on different aspects.

\subsection{Fall and Activity Recognition}

There are several techniques, algorithms and positions of devices for falls and activities recognitions. Leu et al. [5] implemented a fall detection system using a tri-axial accelerometer and gyroscope installed in a mobile phone. Shibuya et al. [6] proposed a lightweight and wearable device to detect falls and six other types of activities using a threshold-based algorithm. Kwon et al. [7] proposed an unsupervised self-learning method for activity recognition with the sensor data collected from a smartphone. A similar research was proposed in [8] presenting a method to recognize a person's activities from the smartphone's sensors using a mixture-of-experts (ME) model with both labeled and unlabeled data. Shawen et al. [9] implemented a classifier for a lower limb amputation where the mobile phone was carried across multiple locations with no predetermined orientation.

\subsection{Devices and Wearables}

The most recent research for falls and activities has been categorized into three main approaches based on visual analysis, ambient context or wearable technology [10].

Vision-based techniques: Vision or camera-based systems provide very rich personal and environmental data for falls detection and activity recognition systems [11]. Ambient-based techniques: Sensors that can capture ambient conditions used for identifying falls through infrared technology, sound detection using microphones, vibration data or a pressure sensor [12]. Wearable-based techniques: A variety of wearable devices are widely popular and commercially available on the market. These devices can provide a wide range of sensor data that can be used to recognize various activities. Moreover, they can be used in various locations both indoor and outdoor but the duration of monitoring is limited by the battery life [13].

\subsection{Health Monitoring Systems}

There is a growing trend to leverage the recent technology advancements and high penetration and ubiquity of smart devices to replace in-hospital health monitoring with a more convenient and cost-efficient mobile health monitoring [14]. Mobile, portable and wearable devices can be efficiently used to track health conditions, activities and relevant environment context. Mobile health monitoring systems are able to better cope with active lifestyle, hence offer more freedom and convenience to patients [15]. In the past few years, most of the proposed research was primary based on cameras, microphones, and other type of embedded devices were used for detecting falls and recognizing a number of daily activities related to wellbeing. However, such devices were cumbersome, obtrusive and difficult to install. It was also inconvenient for regular daily life use, especially for outdoor scenarios or sleeping. 
The proposed research in this paper leverages several good ideas from previous research efforts, but takes mobile health monitoring into the next level of convenience, accuracy and cost-efficiency. We basically use a smartwatch and a number of environment sensors for fall detection and daily activity recognition. This makes it easier and affordable for patients. Our classifier utilizes a number of algorithms to recognize falls and activities to achieve high accuracy. We also provide health risk measures/thresholds in order to alert or warn patients/healthcare providers in case of unfolding abnormal events or high-risk activities. Lastly, several easy-to-understand visualizations are presented to assist patients to better understand their current health conditions and risk levels in real-time.

\section{System Architecture}

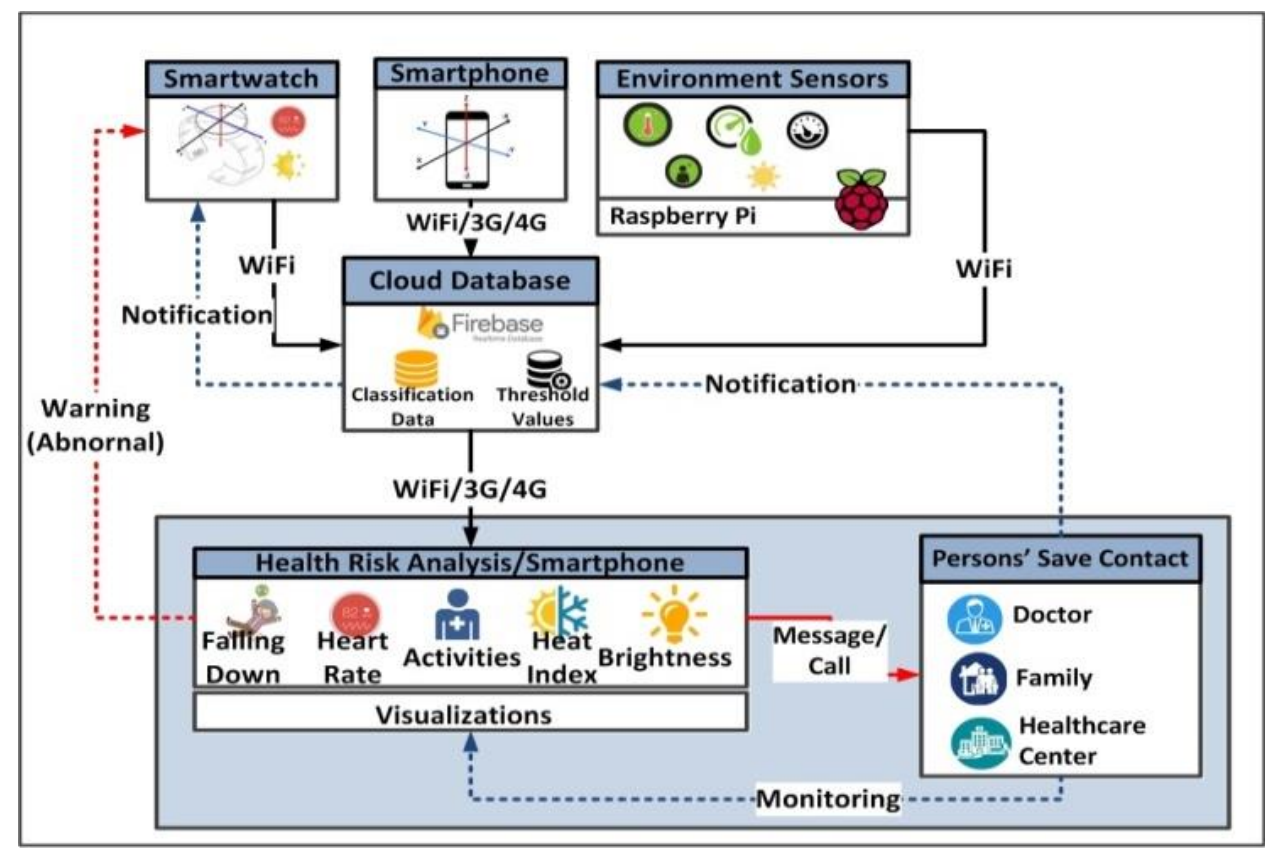

Fig. 1. An abstract architecture of the proposed activity recognition system.

Fig. 1 shows an abstract overview of the proposed activity recognition system. The system supports three core functions: (1) fall detection and activity recognition using machine learning algorithms; (2) health risk analysis and environment data analysis based on personal and environment context; (3) visualization of various activities, surrounding context, and vital signs.

The system reads sensor data from the user's smartwatch (on the wrist) and smartphone. The primary sensor readings are the accelerometer and gyroscope data. iWatch also reads the vital signs available from the smartwatch, depending on the model capabilities. Environment context is captured by a number of sensors that can measure ambient light, temperate, pressure, and humidity. These sensors typically use a gateway to connect to the Internet (e.g., a Raspberry Pi). Our system is flexible and extendable and can automatically discover nearby sensors and integrate their functionality/services using discovery protocols such as CoAP, UPnP and MQTT. Data gathered from all sensors are sent to a cloud-based server for processing and storage. The user interface displays the various activity detected as well as surrounding conditions. The user dashboard is divided into three main sections: current and past personal activities, real-time vital signs (e.g., heart rate), and environment conditions surrounding the user.

A variety of machine learning techniques are used to recognize the user's activity in real-time. Ten common key activities can be detected from the smartwatch data including sitting, standing, lying down, walking, jogging, typing, holding/reading, looking at a watch, waving and falling down. Four activities can be 
captured by data collected from the smartphone including holding, moving, calling and lying down. The data gathered from both the smartwatch and phone can be correlated to improve the overall accuracy. The activity recognition is performed in two phases: training phase and real-time recognition phase.

\subsection{Model Training Phase}

A number of machine learning models have been tested to evaluate their performance with our target application and data set. We train the selected model with our real-time data that we collected from many subjects performing guided activities. The objective of the training phase is to improve the model accuracy specifically to our case. The sequence of activity recognition performed in this phase is shown in Fig. 2 .

\begin{tabular}{|l|}
\hline $\begin{array}{c}\text { 1. Accelerometer Sensor Data Collection } \\
\text { (Time Series Data Stream from devices) }\end{array}$ \\
\begin{tabular}{|c|c|c|}
\hline \multicolumn{3}{|c|}{ 2. Data Pre-Processing } \\
\hline \multicolumn{3}{|c|}{ Min-Max Normalization } \\
\hline \multicolumn{3}{|c|}{ Euclidean Distance } \\
\hline \multicolumn{3}{|c|}{ 3. Model Evaluation } \\
\hline $\begin{array}{c}\text { Random } \\
\text { Forest }\end{array}$ Decision & KNN & Naïve \\
\hline \multicolumn{3}{|c|}{ Tree (J48) } \\
Cross-validation (10-fold)
\end{tabular} \\
\hline \multicolumn{3}{|c|}{ Bayes } \\
\hline \multicolumn{3}{|c|}{ Optimal Classifier } \\
\hline
\end{tabular}

\begin{tabular}{|c|} 
4. Real-Time Fall and Activity Classification \\
\hline Time Series Data : Window Size ( 15 data points) \\
\hline Calculation of Velocity \\
\hline Activity Model \\
\hline $\begin{array}{c}\text { Class of Falls and Activities } \\
\text { (Visualization and Health Risk Analysis) }\end{array}$ \\
\hline
\end{tabular}

Fig. 2. Integrated steps of the proposed activity recognition system.

Data Collection: The system gathers accelerometer, heart rate, and light sensor data from the smartwatch, and only accelerometer data from the smartphone. The tri-axial accelerometer consists of $\mathrm{X}, \mathrm{Y}$ and $\mathrm{Z}$ coordinates as shown in Fig. 3. The smartwatch sensor data were obtained from 30 subjects performing 10 activities while wearing the smartwatch on either left and right wrist. We noticed no difference on either case. The data were continuously collected for 10 seconds at a 0.2 -sec sampling rate. The procedure was repeated three times for both the smartwatch and the smartphone data collection to ensure statistical significance.

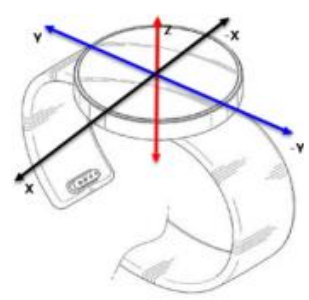

(a)

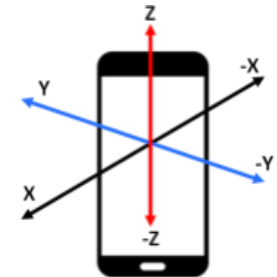

(b)

Fig. 3. (a) Tri-axial accelerometer of smartwatch (b) Tri-axial accelerometer of smartphone. 
Data Pre-Processing: In machine learning, data pre-processing is an important step for data validation, outlier detection, and interpolation of any missing data. The Min-Max normalization standard technique [16] is selected to transform the original data into a range of $[-1,1]$ to adjust the scale and calculate the Euclidean distance for a rate of velocity change as shown in Equation 1.

$$
\operatorname{Difference}(i, j)=\sqrt{(X j-X i)^{2}+(Y j-Y i)^{2}+(Z j-Z i)^{2}}
$$

where Difference $(i, j)$ represents the accelerometer data from the $i^{\text {th }}$ and $j^{\text {th }}$ records, and $X, Y$, and $Z$ represent the three axes.

Model Evaluation: We selected four algorithms to train the data using a 10 -fold cross validation, namely, Random Forest, Decision Tree (J48), Naïve Bayes, and k-Nearest Neighbors. The training data set was collected from 20 subjects performing ten activities.

Table 1. Results of the Activities Testing

\begin{tabular}{|l|l|l|l|l|l|l|}
\hline $\begin{array}{l}\text { Algorithm/ } \\
\text { Activity }\end{array}$ & $\begin{array}{l}\text { Random } \\
\text { Forest }\end{array}$ & $\begin{array}{l}\text { Decision } \\
\text { Tree (J4) }\end{array}$ & $\begin{array}{l}\text { KNN } \\
(\mathrm{k}=3)\end{array}$ & $\begin{array}{l}\text { KNN } \\
(\mathrm{k}=5)\end{array}$ & $\begin{array}{l}\text { KNN } \\
(\mathrm{k}=7)\end{array}$ & $\begin{array}{l}\text { Naïve } \\
\text { Bayes }\end{array}$ \\
\hline Smartwatch'Activity & $99.51 *$ & 99.08 & 99.20 & 99.13 & 99.03 & 87.71 \\
\hline Smartphone's Activity & $99.91 *$ & 99.84 & 99.87 & 99.85 & 99.83 & 99.3 \\
\hline
\end{tabular}

Table 1 shows the performance of the four algorithms. The results show that the Random Forest algorithm outperforms the other three algorithms. Thus, Random Forest will be used for real-time activity recognition for both devices.

\subsection{Real-Time Recognition Process}

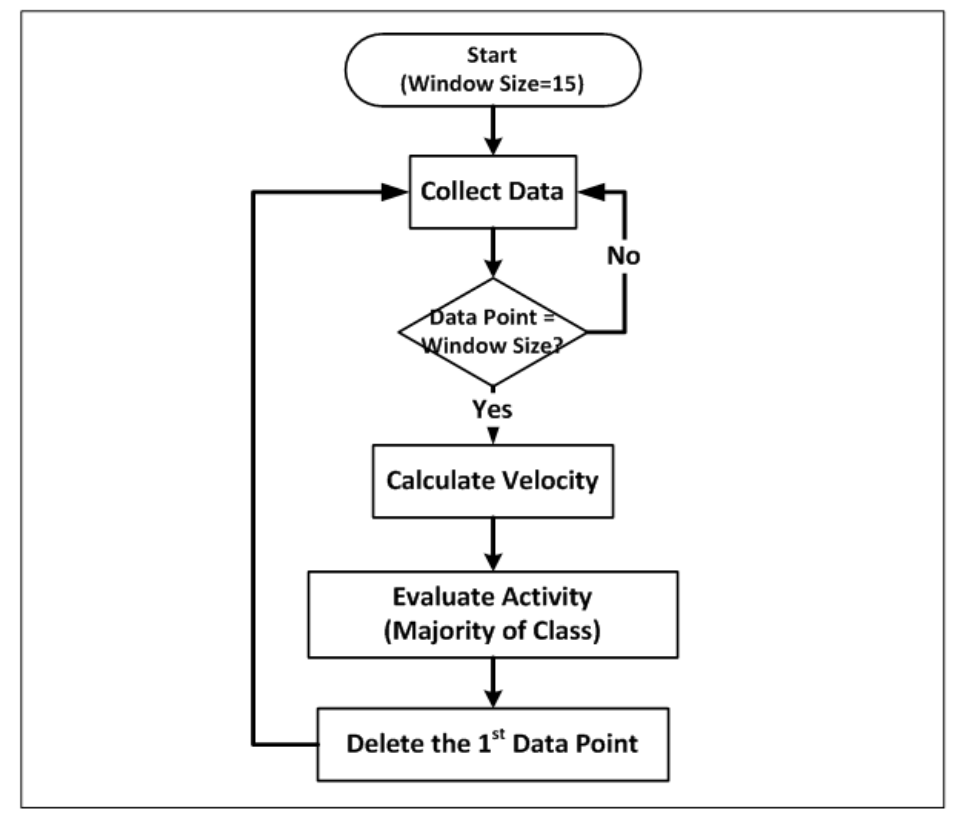

Fig. 4. (a) Real-time classification process.

The real-time recognition process is divided into three sub-processes consisting of data collection, pre-processing and data prediction as shown in Fig. 4. The sensor data is collected and pre-processed similar to the offline training phase for both the smartwatch and the smartphone sensor data. 
Data Gathering: The real-time tri-axial accelerometer sensor data were obtained from 10 human subjects for 10 seconds for each target activities and repeated three times for statistical significance. The total number of obtained data point was $30,000(5 \times 10 \times 3 \times 2 \times 10 \times 10)$ form the smartwatch, and 6,000 $(5 \times 10 \times 3 \times 4 \times 10)$ from the smartphone. We set a window size of 3 seconds buffer time (15 points) to last for one complete data collection cycle.

Runtime Data Pre-processing: Similar to the training phase, data pre-processing was performed at runtime during the recognition phase to ensure high quality of collected data and recognition process.

\section{Experiment Results and Discussion}

In this section, we describe our experimental testbed and setup. We also discuss the findings and provide insights on the overall performance of the proposed system.

\subsection{Experiment Setup}

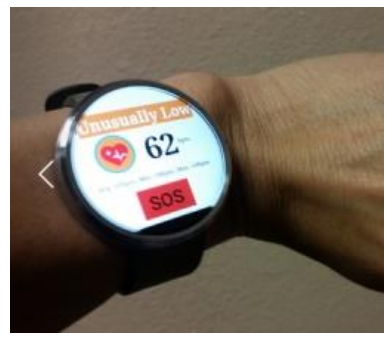

(a)

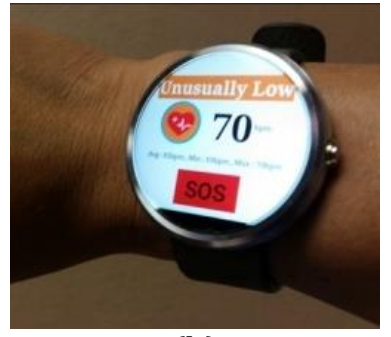

(b)

Fig. 5. (a) Smartwatch on left wrist (b) Smartwatch on the right wrist.

A Moto 360 first generation smartwatch was used for our experiment. The smartwatch ran Android Wear operating system [17]. A Samsung S5 smartphone was connected to the smartwatch to track subject activity.

The subjects tried the smartwatch whether on right and left wrist as shown in Fig. 5 and no significant difference was noticed in the measurements. The smartwatch reported accelerometer readings, heart rate, and ambient light. The smartphone was used to gather the accelerometer sensor data only.

A set of sensors was used to measure the environment parameters including temperature, humidity, pressure [18], light [19], ultrasonic [20], and motion sensors as shown in Fig. 6. In real-life deployments, such sensors can be placed around the areas where the user is mostly expected to occupy such as living room, bedroom, kitchen, garage, etc.

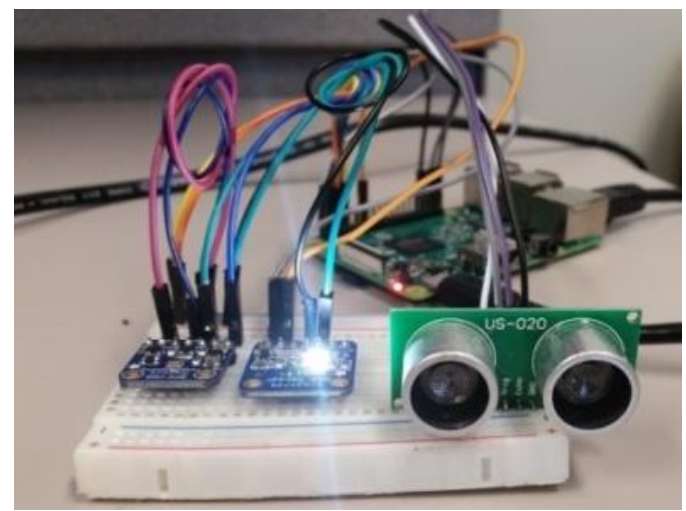

Fig. 6. Environment sensor devices. 


\subsection{Real-Time Classification}

Table 2. Evaluation Results of Real-Time Smartwatch's Activities.

\begin{tabular}{|c|c|c|c|c|c|c|c|c|c|c|c|}
\hline $\begin{array}{l}\text { Activities/ } \\
\text { Evaluation }\end{array}$ & $\stackrel{\infty}{:}$ & 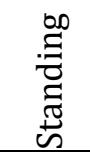 & 茴 & 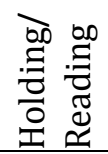 & . & 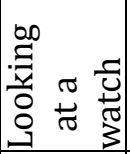 & 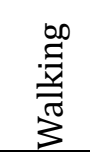 & 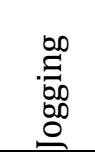 & 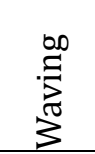 & 先 & 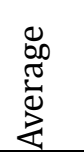 \\
\hline Accuracy (\%) & 100.0 & 100.0 & 96.32 & 100.0 & 96.32 & 100.0 & 100.0 & 100.0 & 99.30 & 100.0 & 99.19 \\
\hline Precision & 1.000 & 1.000 & 0.963 & 1.000 & 0.963 & 1.000 & 1.00 & 1.000 & 0.930 & 1.000 & 0.985 \\
\hline Recall & 1.000 & 1.000 & 0.963 & 1.000 & 0.963 & 1.000 & 1.000 & 0.935 & 1.000 & 1.000 & 0.986 \\
\hline
\end{tabular}

Table 3. Evaluation Results of Real-Time Smartphone's Activities.

\begin{tabular}{|l|l|l|l|l|l|}
\hline $\begin{array}{l}\text { Activities/ } \\
\text { Evaluation }\end{array}$ & Holding & Putting & Calling & Moving & Average \\
\hline Accuracy (\%) & 100.00 & 100.00 & 100.00 & 100.00 & 100.00 \\
\hline Precision & 1.000 & 1.000 & 1.000 & 1.000 & 1.000 \\
\hline Recall & 1.000 & 1.000 & 1.000 & 1.000 & 1.000 \\
\hline
\end{tabular}

The Random Forest classification algorithm achieved an average of 99.19 percent accuracy, precision at 0.985 and recall at 0.986 for smartwatch-based activity recognition as shown in Table 2, and 100 percent for smartphone-based activity recognition as shown in Table 3.

\subsection{Fall and Activity Visualizations}

The mobile application provides a rich interactive graphical interface that uses simple representative symbols to display to the user the current activities and vital signs including, heart rate, the most recent three activities detected by the system. The dashboard also provides a communication platform that connects users with their healthcare providers.

\subsubsection{User dashboard}

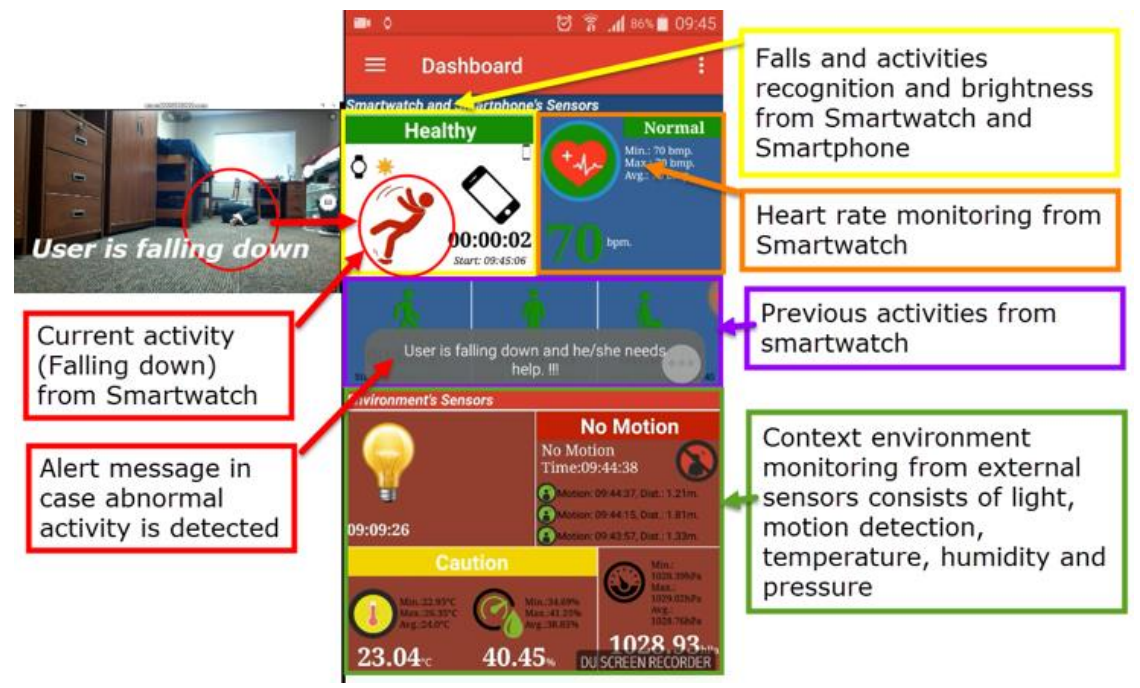

Fig. 7. Dashboard of activity and environment monitoring.

Fig. 7 shows the end user dashboard divided into primarily two sections. The top section shows current and recent activities and vital signs. The bottom section displays the environment context around the user including light, temperature, humidity, and pressure. These surrounding conditions may pose some health risks to the user/patient or determine the context when something happens for better assessments. 


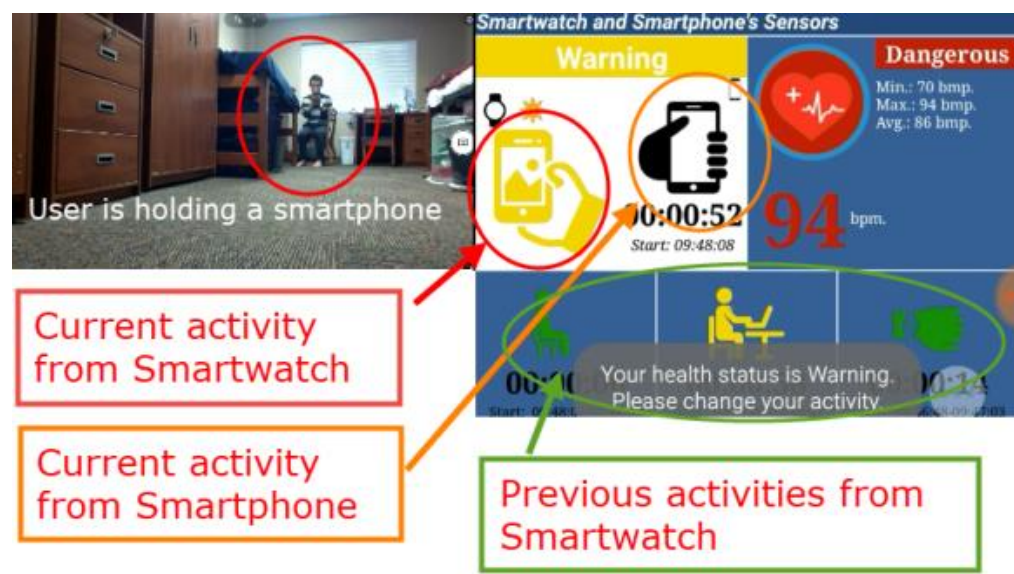

Fig. 8. The current and previous activities from smartwatch and smart phone.

Fig. 8 shows the current activity, elapsed time during the activity and visual representation of the associated health risks. The system also provides the brightness level surrounding the user, measured by the smartwatch to help user adjust the brightness level for current activity (e.g., reading) to maintain healthy eyesight [21].

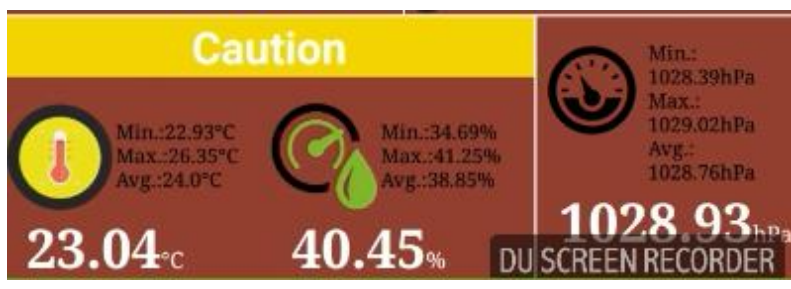

Fig. 9. Environment monitoring and warning system.

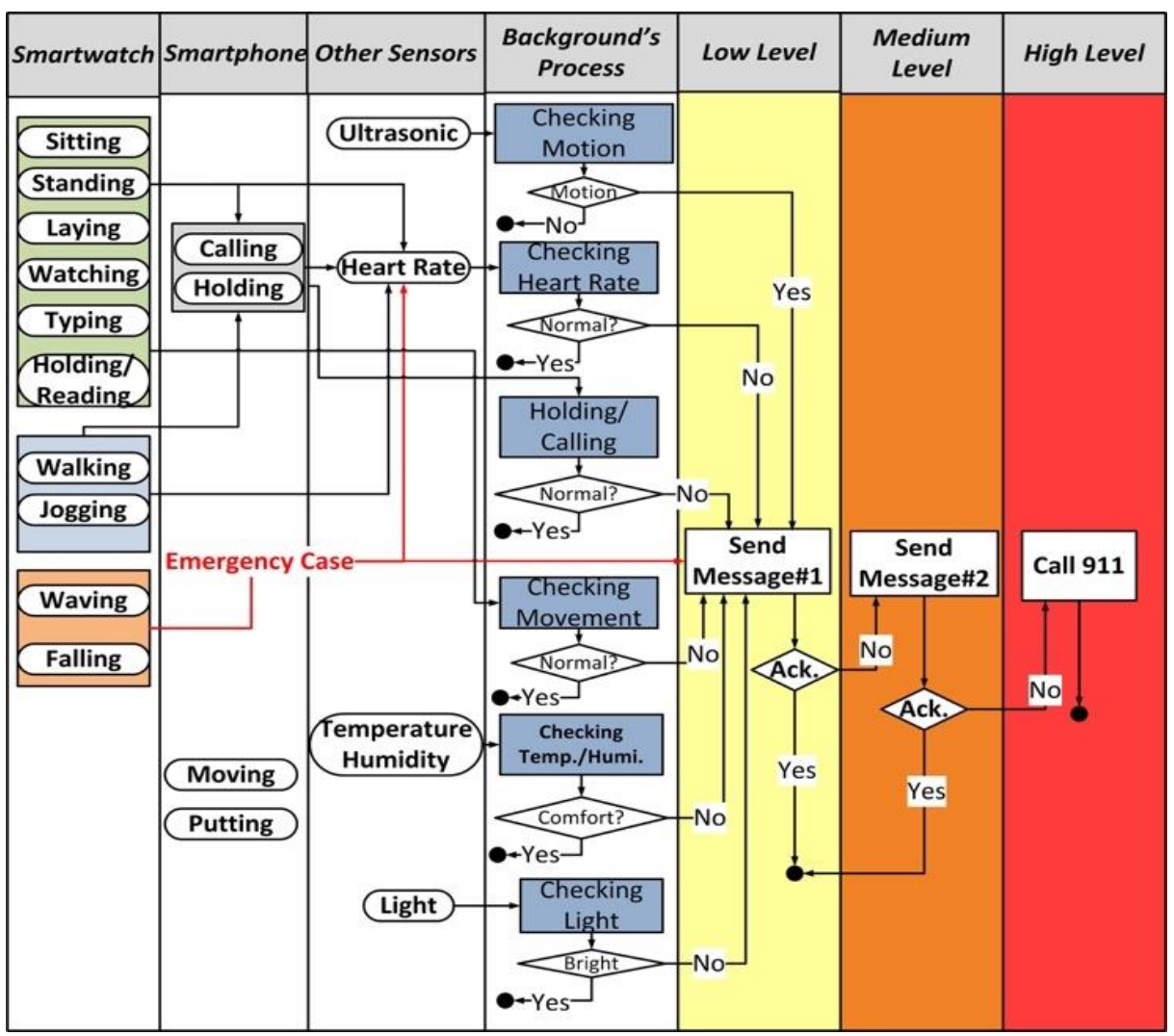

Fig. 10. The warning system flow. 
The bottom part of the dashboard displays the environment context around the user such as light, temperature, humidity and pressure in order to help the user to avoid uncomfortable area. The system provides the functionality to identify the level of health risk based on pre-specified thresholds for temperature and humidity. The normal level is represented by the green color; caution level is yellow, and dangerous level is red [22] as shown in Fig. 9.

\subsubsection{Health risk analysis}

The system performs background analytics based on the raw sensor data gathered from personal and environment sensors to provide warnings and recommendations related to the user's health conditions. Four thresholds refer to risk level: normal, low risk, moderate risk, and high risk, and health risk analysis is shown in Fig. 10.

\subsubsection{Activity reporting}

The system provides visualized activity report based on historical data using various visualization techniques including pie chart, graphs, bars, etc. These reports provide an interaction visual understanding of the user's past activities including health risk level and percentage.

Fig. 11 shows two sample reports, one for the activity summary (Fig. 11.a) and another for heart rate (Fig. 11.b). Fig. 11.b shows a pie chart at the bottom that represents the summary heart rate risk level. The heart rate status is indicated by different colors [23]: green means healthy, yellow represents a caution level, orange color indicates unhealthy level, and red means risky level.

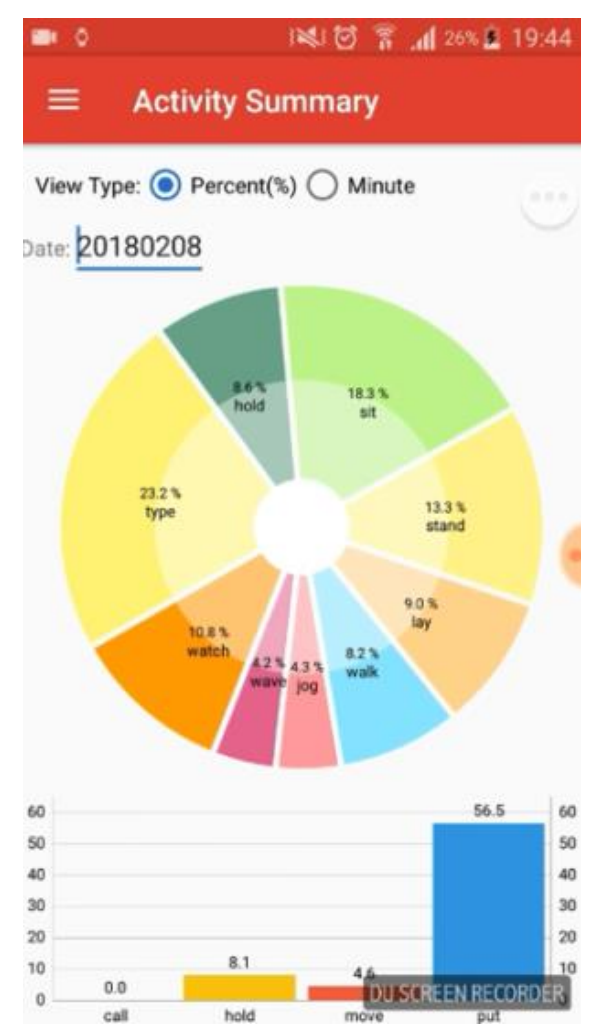

(a)

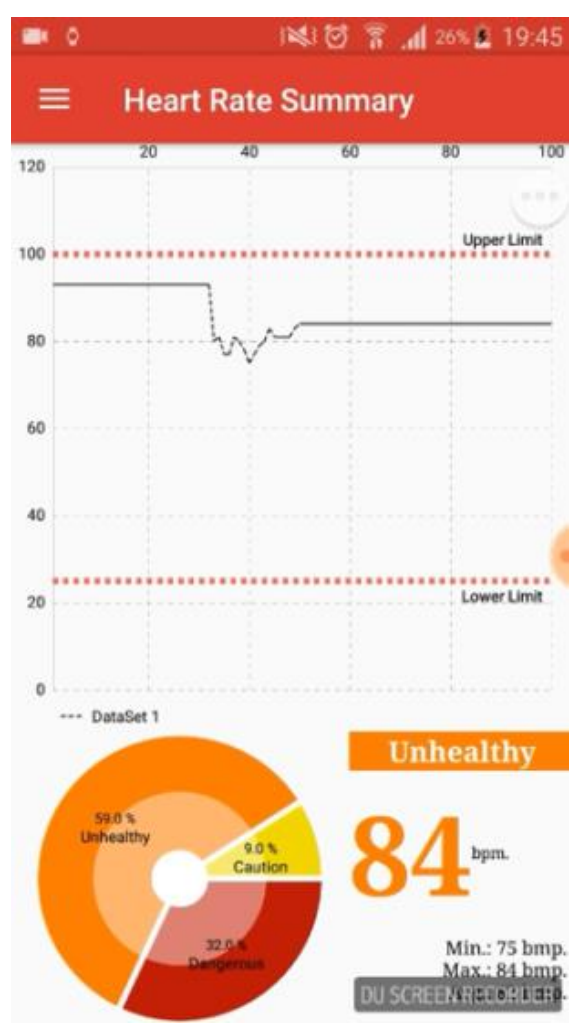

(b)

Fig. 11. (a) Summary activity report by date (b) Summary of heart rate.

The system also offers real-time heart rate monitoring during the current activity, and it can show the changing heart rate of the subject while performing the activity as shown in Fig. 12. Furthermore, the system provides a recommendation on the upper and lower boundaries of healthy limits, and it can be personalized for each user through watching the user's activities. 


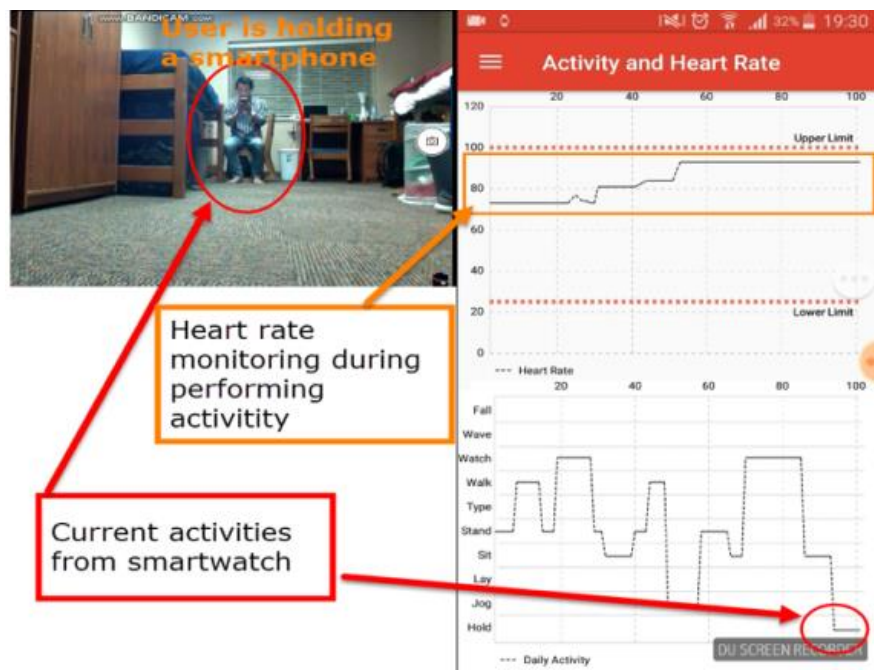

Fig. 12. Heart rate monitoring during activities.

\subsubsection{Min-max activity recommendation}

It is always beneficial for users to know their limits for various activities. The system offers radar graphs with healthy borderlines to monitor and track health risk levels for each of the seven activities (sitting, standing, lying down, walking, jogging, typing, and holding/reading) not including three activities (fall, wave and look at the watch) because the duration of these activities is too short which makes it difficult to measure using the same units.

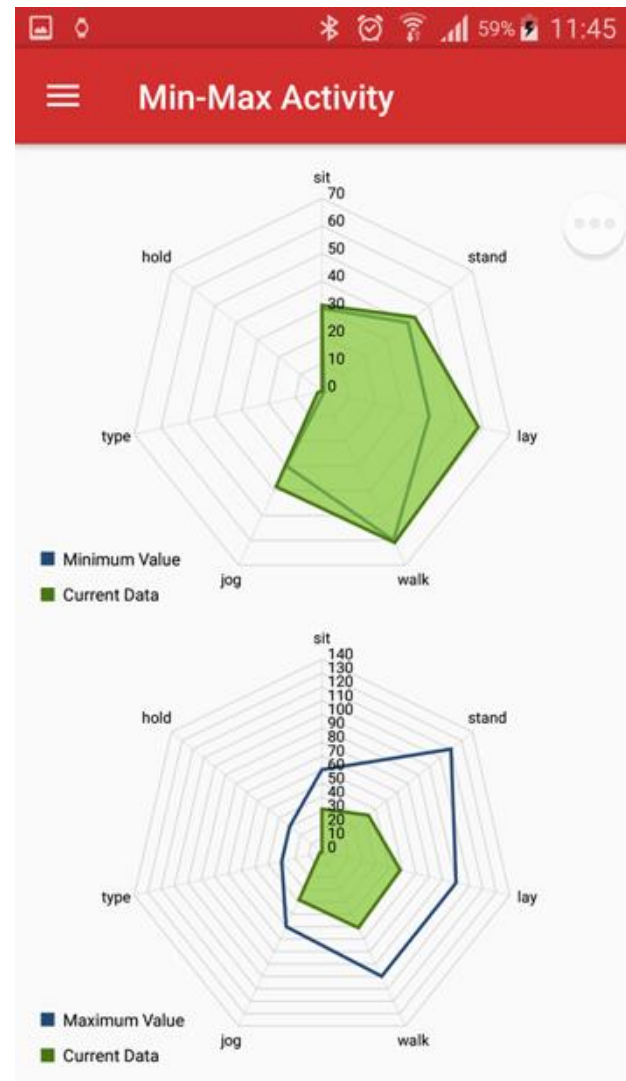

(a)
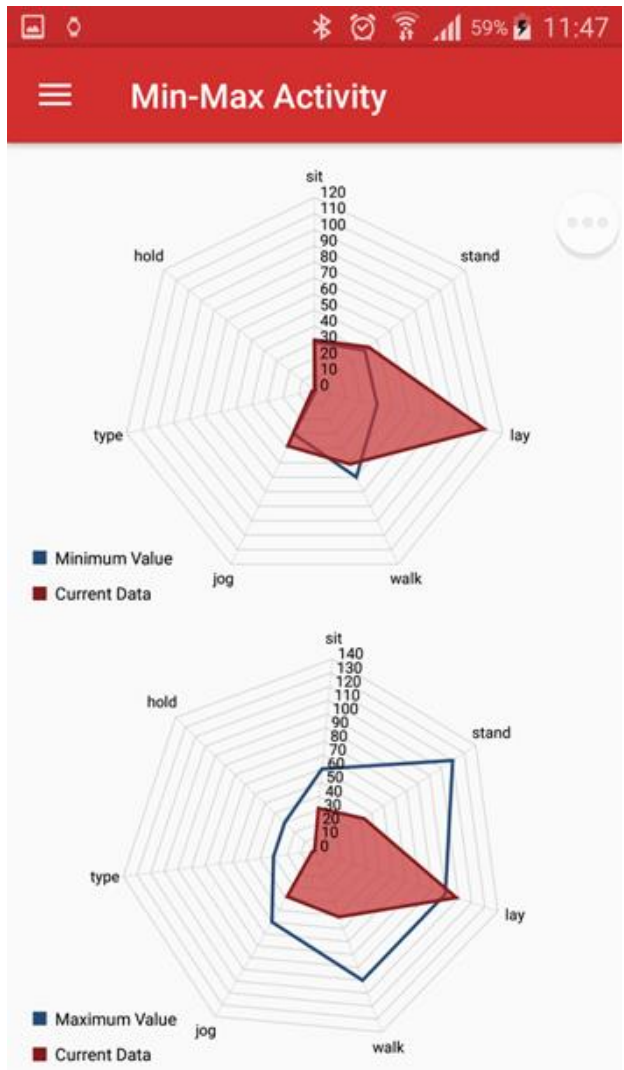

(b)

Fig. 13. Radar graphs of recommended daily activities (minimum and maximum values): (a) Healthy, (b) Unhealthy. 
The Min-Max activity threshold values will be determined by caregivers and will be configured in the system. These values will be set as the base line for each activity which are processed in the background.

Fig. 13 shows the recommended minimum and maximum values of the total daily minutes (blue border) for each activity and the color of the radar graph represents the risk level. Green means healthy, and red means unhealthy as shown in Fig. 13.b. Threshold values are typically personal and must be set for individuals by healthcare providers based on health status, habits, and personal fitness level.

In the event of falling down, the SOS will be shown immediately on the smartwatch screen, and the user can hit the button in order to request an emergency assistance as shown in Fig. 14. In neither cases, the system automatically places an emergency call within 10 seconds of the user falling and provides the user's current location.

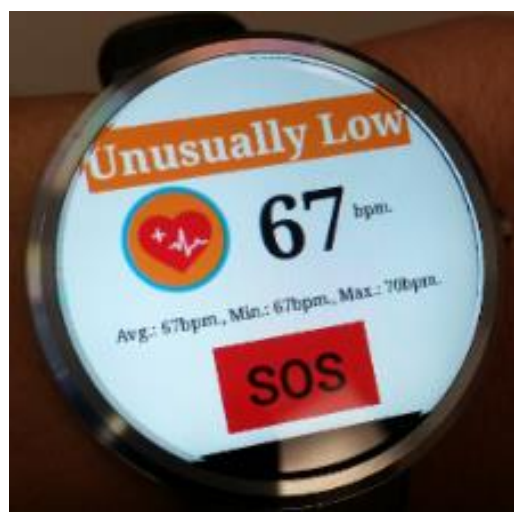

Fig. 14. SOS button on the smartwatch.

\section{Conclusion and Future Work}

In this paper, we present iWatch, a practical and affordable fall and daily activities recognition system using a smartwatch and a smartphone for indoor and outdoor settings. Real-time sensor data are collected from the user's smartwatch and smartphone as well as from sensors deployed in the surrounding environment. A number of machine learning techniques have been implemented and tested to build an accurate and lightweight classifier model for fall detection and activity recognition. iWatch demonstrates good performance with an overall $99.19 \%$ accuracy, 0.985 precision, and 0.986 recall using smartwatch sensor data. The trained classifier shows a perfect $100 \%$ accuracy, and 1.00 on both precision and recall for smartphone sensor data.

iWatch dashboard presents recognized activities and vital signs to the user using simple visualizations. It also provides warnings when health risk levels or vital signs exceed pre-specified limits. Environment context is also presented for guided assistance and better assessments in post-event evaluation.

\section{Acknowledgment}

We would like to acknowledge Data Science and Engineering Laboratory (D-Lab) of School of Information Technology at King Mongkut's University of Technology Thonburi (KMUTT) and School of Computing \& Informatics at University of Louisiana at Lafayette (UL). The first author has received a financial support from the KMUTT's Petchra Pra Jom Klao Research Scholarship. We also acknowledge the partial support from the Innovation Hubs project under the Council of University Presidents of Thailand (CUPT). We wish to thank all the volunteers for helping with the data collection. This work passed the institutional review board (IRB) process of School of Liberal Arts at KMUTT in December 2015. 


\section{References}

[1] Stepler, R. (2016). Smaller share of women ages 65 and older are living alone: More are living with spouse or children. Pew Research Center. Retrieved from http://www.pewsocialtrends.org/2016/02/18/smaller-share-of-women-ages-65-and-older-are-living -alone/

[2] Centers for Disease Control and Prevention. (2012). Web-based injury statistics query and reporting system (wisqars). Atlanta, GA: National Center for Injury Prevention and Control. Retrieved from http://www.cdc.gov/homeandre -creational safety/ falls/adultfalls.html

[3] National Safety Council. (2017). Top Causes of Unintentional Injury and Death in Homes and Communities. Retrieved from http://www.nsc.org/learn/safety-knowledge/Pages/ safety-at-home.aspx

[4] Barlow, J., Singh, D., Bayer, S., \& Curry, R. (2007). A systematic review of the benefits of home telecare for frail elderly people and those with long-term conditions. Journal of Telemedicine and Telecare, 13(4), 172-179.

[5] Leu, F. Y., Ko, C. Y., Lin, Y. C., Susanto, H., \& Yu, H. C. (2017). Fall detection and motion classification by using decision tree on mobile phone. Smart Sensors Networks (pp. 205-237).

[6] Shibuya, N., Nukala, B. T., Rodriguez, A. I., Tsay, J., Nguyen, T. Q., Zupancic, S., \& Lie, D. Y. (2015, January). A real-time fall detection system using a wearable gait analysis sensor and a support vector machine (svm) classifier. Proceedings of 2015 Eighth International Conference on Mobile Computing and Ubiquitous Networking (ICMU) (pp. 66-67).

[7] Kwon, Y., Kang, K., \& Bae, C. (2014). Unsupervised learning for human activity recognition using smartphone sensors. Expert Systems with Applications, 41(14), 6067-6074.

[8] Lee, Y. S., \& Cho, S. B. (2014). Activity recognition with android phone using mixture-of-experts co-trained with labeled and unlabeled data. Neurocomputing, 126, 106-115.

[9] Shawen, N., Lonini, L., Mummidisetty, C. K., Shparii, I., Albert, M. V., Kording, K., \& Jayaraman, A. (2017). Fall detection in individuals with lower limb amputations using mobile phones: Machine learning enhances robustness for real-world applications. JMIR mHealth and uHealth, 5(10).

[10] Mubashir, M., Shao, L., \& Seed, L. (2013). A survey on fall detection: Principles and approache. Neurocomputing, 100, 144-152.

[11] Ozcan, K., \& Velipasalar, S. (2016). Wearable camera-and accelerometer-based fall detection on portable devices. IEEE Embedded Systems Letters, 8(1), 6-9.

[12] Droghini, D., Ferretti, D., Principi, E., Squartini, S., \& Piazza, F. (2017). A combined one-class SVM and template-matching approach for user-aided human fall detection by means of floor acoustic features. Computational Intelligence and Neuroscience.

[13] Martín, H., Bernardos, A. M., Iglesias, J., \& Casar, J. R. (2013). Activity logging using lightweight classification techniques in mobile devices. Personal and ubiquitous computing, 17(4), 675-695.

[14] Fajingbesi, F. E., Olanrewaju, R. F., Pampori, B. R., Khan, S., \& Yacoob, M. (2017). Real time telemedical health care systems with wearable sensors. Asian Journal of Pharmaceutical Research and Health Care, 9(3), 138-144.

[15] Helbostad, J. L., Vereijken, B., Becker, C., Todd, C., Taraldsen, K., Pijnappels, M., \& Mellone, S. (2017). Mobile health applications to promote active and healthy ageing. Sensors, 17(3), 622.

[16] Han, J., Pei, J., \& Kamber, M. (2011). Data Mining: Concepts and Techniques. Elsevier.

[17] Motorola Mobility Company. Moto $360 \quad$ (1st Gen). Retrieved from https://motorola-mobility-enin.custhelp.com/app/home/device /motorola/moto-360-1st-gen 
[18] Adafruit. Adafruit BME280 I2C or SPI Temperature Humidity Pressure Sensor. Retrieved from https://www.adafruit.com/product/2652

[19] Adafruit. RGB Color Sensor with IR Filter and White LED - TCS34725. Retrieved from https://www.adafruit.com/ product/1334

[20] Canada Robotix. US-020 Ultrasonic Ranging Sensor. Retrieved from http://www.canadarobotix.com/sound-microphone/us020-ultrasonic-ranging-sensor

[21] Harvard Health Publishing. Safeguarding Your Sight. Retrieved from https://www.health.harvard.edu/healthbeat/safeguarding-your-sight

[22] Opitz-Stapleton, S., Sabbag, L., Hawley, K., Tran, P., Hoang, L., \& Nguyen, P. H. (2016). Heat index trends and climate change implications for occupational heat exposure in Da Nang, Vietnam. Climate Services, 2, 41-51.

[23] American Heart Association. Target Heart Rates. Retrieved from http://www.heart.org/HEARTORG/HealthyLiving/PhysicalActivity/Target-HeartRates_UCM_434341_Ar ticle.jsp\#. WpDuMKjwZdh
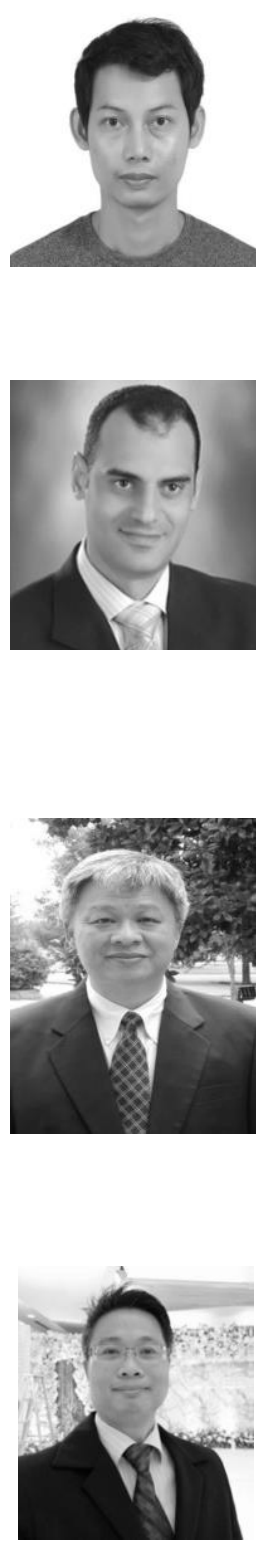

Pornchai Mongkolnam received a Ph.D. degree in computer science from Arizona State University, USA in 2003 and currently works at School of Information Technology (SIT) at King Mongkut's University of Technology Thonburi, Thailand. He is the head of Data Science and Engineering Laboratory (D-Lab) at SIT. 
Chakarida Nukoolkit received a Ph.D. degree in computer science from the University of Alabama, USA in 2001. She is now an assistant professor with the School of Information Technology, King Mongkut's University of Technology Thonburi, Thailand. Her current research interests include data mining, visualization, and artificial intelligence. 